\title{
ON HARDY'S INEQUALITY IN WEIGHTED REARRANGEMENT INVARIANT SPACES AND APPLICATIONS. I
}

\author{
LECH MALIGRANDA
}

\begin{abstract}
We give inequalities relating the norm of a function and the norm of its average operators $P_{\psi}, Q_{\psi}$ and $S_{\psi}, T_{\psi}$ in weighted rearrangement invariant spaces $E_{\kappa . \delta}$ and $E(\mu), d \mu(t)=\tau^{\prime}(t) d t$. These average operators include, for example, the integral mean, the $P_{p}, Q_{p}$ operators of Boyd [4] and Butzer and Feher [6], the average operators $P_{\varphi}, Q_{\varphi}$ and $S_{E}, T_{E}$ from $[14,15,16]$. In the particular case, for some $\psi, \kappa, \delta, \tau$ and $E$ these inequalities were obtained by many authors and applied to a study of interpolation operators and imbedding theorems for Sobolev weight spaces.
\end{abstract}

Let $\psi$ be a positive nondecreasing continuous function on $\mathrm{I}=(0, l), 0<l \leqslant \infty$. We define the average operators $P_{\psi}, Q_{\psi}$ and (if additionally, $\psi$ is an increasing absolutely continuous function with $\left.\psi\left(0^{+}\right)=\lim _{t \rightarrow 0^{+}} \psi(t)=0\right) S_{\psi}, T_{\psi}$, namely

$$
\begin{aligned}
& \left(P_{\psi} x\right)(t):=\psi(t)^{-1} \int_{0}^{t} x(s) \psi(s) s^{-1} d s, \\
& \left(Q_{\psi} x\right)(t):=\psi(t)^{-1} \int_{t}^{l} x(s) \psi(s) s^{-1} d s, \\
& \left(S_{\psi} x\right)(t):=\psi(t)^{-1} \int_{0}^{t} x(s) \psi^{\prime}(s) d s, \\
& \left(T_{\psi} x\right)(t):=\psi(t)^{-1} \int_{t}^{l} x(s) \psi^{\prime}(s) d s
\end{aligned}
$$

whenever the required integral exists for almost all $t \in I$. For $\psi(t)=t^{a}, 0<a<\infty$, we write $P_{a}, Q_{a}$ and $S_{a}, T_{a}$, instead of $P_{\psi}, Q_{\psi}$ and $S_{\psi}, T_{\psi}$, respectively.

We will investigate the boundedness of operators (1)-(4) in weighted rearrangement invariant spaces with the aid of indices.

The lower index $p^{\prime}(\psi)$ and the upper index $q^{\prime}(\psi)$ of a positive measurable function $\psi$ on $I$ are defined in terms of the submultiplicative function on $(0, \infty)$,

$$
\begin{aligned}
M^{\prime}(s, \psi) & =\sup _{t \in I, t s \in I} \frac{\psi(t s)}{\psi(t)}=\sup _{t \in(0, \min (1,1 / s) l)} \frac{\psi(t s)}{\psi(t)}: \\
p^{\prime}(\psi) & =\lim _{s \rightarrow 0^{+}} \frac{\ln M^{\prime}(s, \psi)}{\ln s},
\end{aligned}
$$

Received by the editors June 1, 1981 and, in revised form, March 20, 1982.

1980 Mathematics Subject Classification. Primary 26A86, 46E30.

Key words and phrases. Average operator, Hardy inequality, rearrangement invariant spaces, indices 


$$
q^{\prime}(\psi)=\lim _{s \rightarrow \infty} \frac{\ln M^{\prime}(s, \psi)}{\ln s}
$$

(see $[16,10])$.

REMARK 1. We shall consider only $\psi$ such that limits (5), (6) exist. We see that this is meaningful if $M^{I}(s, \psi)$ is a finite measurable function on $(0, \infty)$ or $M^{I}(s, \psi)$ is a monotone function on $(0, \infty)$.

We assume that $E=E(m)$ is an ideal Banach function space of measurable (equivalence classes of Lebesgue measurable) functions on $I=(0, l), 0<l \leqslant \infty$, which norm $\|\cdot\|_{E}$ is rearrangement invariant in the sense that two functions which are equimeasurable with respect to $m$ have the same norm. We called this space a rearrangement invariant space (r.i. space) or symmetric space in the terminology of Semenov (for more details, see [10]).

Examples of r.i. spaces include the Lebesgue $L^{p}$-spaces, the Orlicz $L^{F}$-spaces, the Lorentz spaces $\Lambda, M, L(p, q)$ and the Lorentz-Orlicz spaces $L\left(\varphi, L^{F}\right), \Lambda\left(\varphi, L^{F}\right)$. Also if $E, F$ are r.i. spaces, so are $E \cap F, E+F$ and $\vec{A}^{E}$, where $A$ is symmetric linear subset of $E$ ( $A$ is symmetric if $x \in A$ and $y^{*}(t) \leqslant x^{*}(t)$ imply $y \in A$ ).

If $X$ and $Y$ are (quasi-)Banach spaces, $[X, Y]$ will denote the space of all bounded linear operators from $X$ into $Y$. We abbreviate $[X, X]=[X]$.

The lower index $\alpha^{\prime}(E)$ and the upper index $\beta^{\prime}(E)$ corresponding to the r.i. space $E$ on $I$ were defined by Boyd [4] in terms of the submultiplicative function $h(s, E)$, where, for r.i. space $E, h(s, E)$ denotes the norm in $[E]$ of the dilation operator $\sigma_{s}$, $\left(\sigma_{s} x\right)(t)=x(t / s)$ if $t / s \leqslant l,\left(\sigma_{s} x\right)(t)=0$ if $t / s>l$ :

$$
\begin{aligned}
& \alpha^{\prime}(E)=\lim _{s \rightarrow 0^{+}} \frac{\ln h(s, E)}{\ln s}, \\
& \beta^{\prime}(E)=\lim _{s \rightarrow \infty} \frac{\ln h(s, E)}{\ln s} .
\end{aligned}
$$

For the various r.i. spaces $E$ and functions $\psi$ the indices were computed by Boyd [5] and the author $[15,16]$.

The weighted r.i. space $E_{\kappa, \delta}$, where $\kappa$ is a positive measurable function on $I$ and $\delta$ : $I \rightarrow I$ is a measurable function, consists of those measurable functions $x$ on $I$ for which $x \circ \delta$ is measurable and

$$
\|x\|_{E_{x, \delta}}=\|\kappa(t) x(\delta(t))\|_{E}<\infty .
$$

Let us denote by $E_{\kappa, \delta}^{(*)}$ the set of all measurable functions $x$ on $I$ for which $\kappa(t) x^{*}(\delta(t)) \in E$. The set $E_{\kappa, \delta}^{(*)}$ is not a Banach space in general (functional $\left\|\kappa(t) x^{*}(\delta(t))\right\|_{E}$ does not define a norm, since the triangle inequality fails); therefore we introduce the r.i. space $E_{\kappa, \delta}^{(* *)}$ with the norm

$$
\|x\|_{E_{k, \delta}^{(* *)}}=\left\|\kappa(t) x^{* *}(\delta(t))\right\|_{E}, \quad \text { where } x^{* *}(t)=\frac{1}{t} \int_{0}^{t} x^{*}(s) d s .
$$

REMARK 2. One has

$$
E_{\kappa, \delta}^{(* *)} \subset E_{\kappa, \delta}^{(*)}
$$


with a continuous inclusion map. If equality in (11) holds, then $E_{\kappa, \delta}^{(*)}$ can be renormed equivalently.

EXAMPLE 1. Let $\tau: I \rightarrow I$ be an increasing, absolutely continuous function with $\tau(I)=I$. For any measurable set $A \subset I$ we define measure by

$$
\mu(A)=\int_{A} \tau^{\prime}(t) d t .
$$

The weighted r.i. space $E(\mu)$ then consists of those $\mu$-measurable functions on $I$ for which $x_{\mu}^{*} \in E(m)$ and the norm in $E(\mu)$ is given by $\|x\|_{E(\mu)}=\left\|x_{\mu}^{*}\right\|_{E}$. Here, as usual, $x_{\mu}^{*}$ denotes the nonnegative, nonincreasing rearrangement of $|x|$ which is equimeasurable with $|x|$ in the sense that

$$
\mu\{t \in I:|x(t)|>a\}=m\left\{t \in I: x_{\mu}^{*}>a\right\}
$$

for all $a>0$.

Proposition 1. $E(\mu)=E_{1, \tau^{-1}}$ with equal norms.

Proof. First, we prove that, for any measurable nonnegative function $x$ on $I$,

$$
\mu\{t \in I: x(t)>a\}=m\left\{t \in I: x\left(t^{-1}(t)\right)>a\right\}
$$

for all $a>0$.

For any measurable set $A \subset I$ we have $\mu(A)=m(\tau(A)$ ) (see [13, p. 174]). Applying this to

$$
\begin{aligned}
& A=\{t \in I: x(t)>a\}=x^{-1}(a, \infty)=\tau^{-1}\left[\tau\left(x^{-1}(a, \infty)\right)\right]=\tau^{-1}(B), \\
& B=\tau\left(x^{-1}(a, \infty)\right)=\left\{t \in I: x\left(\tau^{-1}(t)\right)>a\right\}
\end{aligned}
$$

we get

$$
\mu(A)=m\left(\tau\left(\tau^{-1}(B)\right)\right)=m(B) .
$$

From (14), we have, for all $a>0$,

$$
m\left\{t \in I: x_{\mu}^{*}>a\right\} \stackrel{(13)}{=} \mu\{t \in I:|x(t)|>a\} \stackrel{(14)}{=} m\left\{t \in I:\left|x\left(\tau^{-1}(t)\right)\right|>a\right\} .
$$

Hence $\left\|x_{\mu}^{*}\right\|_{E}=\left\|x \circ \tau^{-1}\right\|_{E}$, i.e.

$$
\|x\|_{E(\mu)}=\left\|x_{\mu}^{*}\right\|_{E}=\left\|x \circ \tau_{\varphi}^{-1}\right\|_{E}=\|x\|_{E_{1}, \tau^{-1}} .
$$

EXAMPLE 2. Let $1 \leqslant q<\infty$ and let $\varphi$ be a positive, nondecreasing, concave function on $I$ such that $\varphi\left(0^{+}\right)=0$. Then $L_{\varphi^{(}(t)^{1 / q}, t}^{q(*)}=\Lambda(\varphi, q)$ and $L_{t^{-1 / q_{\varphi}}(t), t}^{q(*)}=$ $L(\varphi, q)$, where

$$
\begin{aligned}
\|x\|_{\Lambda(\varphi, q)} & =\left(\int_{0}^{l} x^{*}(t)^{q} \varphi^{\prime}(t) d t\right)^{1 / q}, \\
\|x\|_{L(\varphi, q)} & =\left(\int_{0}^{l}\left[\varphi(t) x^{*}(t)\right]^{q} d t / t\right)^{1 / q} .
\end{aligned}
$$


Note that $\|\cdot\|_{\Lambda(\varphi, q)}$ satisfies the triangle inequality and $\|\cdot\|_{L(\varphi, q)}$ satisfies the triangle inequality iff $t^{-1 / 9} \varphi(t)$ is equivalent to a nonincreasing function (Lorentz [12]). However, if $q^{\prime}(\varphi)<1$ then in $L(\varphi, q)$ an equivalent norm can be introduced (see Proposition 2).

LeMma 1 [10, p.136]. If $E$ is a r.i. space on $I, \varphi$ a nondecreasing continuous function, and $u$ a nonnegative measurable function on $(a, b), 0 \leqslant a<b \leqslant \infty$, then, for all $x \in E$,

$$
\left\|\int_{a}^{b} \sigma_{1 / s} x^{*}(t) u(s) d \varphi(s)\right\|_{E} \leqslant \int_{a}^{b}\left\|\sigma_{1 / s} x^{*}(t)\right\|_{E} u(s) d \varphi(s) .
$$

For a positive nondecreasing function $f$ defined on $I$ and for ri. space $E$ on $I$ we denote

$$
\begin{gathered}
0 \leqslant a_{f} \leqslant t f^{\prime}(t) / f(t) \leqslant b_{f} \leqslant \infty \quad \text { a.e. on } I, \\
C_{0}(E, f)=\int_{0}^{1} h\left(s^{-1}, E\right) M^{\prime}(s, f) d s / s .
\end{gathered}
$$

THeOREM 1. Let $E$ be a r.i. space on $I$ and let $\psi: I \rightarrow \mathbf{R}_{+}$be a nondecreasing continuous function, $\delta: I \rightarrow I$ a nondecreasing absolutely continuous function, and $\kappa:$ $I \rightarrow \mathbf{R}_{+}$a measurable function such that $\delta\left(0^{+}\right)=0$ and $p^{l}(\psi \circ \delta / \kappa)>0$.

(a) If $b_{\delta}<\infty$, E has Fatou norm and $\beta^{\prime}(E)<p^{\prime}(\psi \circ \delta / \kappa)$, then $P_{\psi} \in\left[E_{\kappa, \delta}\right]$ and $\left\|P_{\psi}\right\| \leqslant b_{\delta} C_{0}(E, \psi \circ \delta / \kappa)$.

(b) If $b_{\delta}<\infty$ and $\beta^{\prime}(E)<\min \left\{1, p^{\prime}(\psi \circ \delta / \kappa)\right\}$, then $P_{\psi} \in\left[E_{\kappa, \delta}\right]$ and $\left\|P_{\psi}\right\| \leqslant$ $b_{\delta} C_{0}(E, \psi \circ \delta / \kappa) C_{0}(E$, id $)$.

(c) If $a_{\delta}>0, \psi \circ \delta / \kappa$ is a nondecreasing function, E has Fatou norm and $P_{\psi} \in\left[E_{\kappa, \delta}\right]$, then $\beta^{\prime}(E)<q^{\prime}(\psi \circ \delta / \kappa)$.

Proof. Since $\left(P_{\psi} x\right)(\delta(t)) \leqslant b_{\delta} P_{\psi \circ \delta}(x \circ \delta)(t)$ for almost all $t \in I$ it is sufficient to prove (a) and (b) for $\delta(t)=t$.

(a) See [15, p. 408].

(b) (See Pavlov [19] for $P_{1}$.) We have

$$
\begin{aligned}
\left(\kappa(t) P_{\psi} x(t)\right)^{*} & \leqslant\left(\kappa(t) P_{\psi} x(t)\right)^{* *}=t^{-1} \sup _{m e=t} \int_{e}\left|\kappa(u) P_{\psi} x(u)\right| d u \\
& \leqslant t^{-1} \int_{0}^{1}\left[\sup _{m e=t} \int_{e}|x(u s)| \kappa(u) \frac{\psi(u s)}{\psi(u)} d u\right] d s / s \\
& =\int_{0}^{1}[x(t s) \kappa(t) \psi(t s) / \psi(t)]^{* *} d s / s \\
& \leqslant \int_{0}^{1}\left[\sigma_{1 / s}(x \kappa)(t)\right]^{* *} M^{\prime}(s, \psi / \kappa) d s / s \\
& =\int_{0}^{1} \sigma_{1 / s}\left[(x \kappa)^{* *}(t)\right] M^{\prime}(s, \psi / \kappa) d s / s .
\end{aligned}
$$

From Lemma 1 follows

$$
\left\|\kappa(t) P_{\psi} x(t)\right\|_{E} \leqslant \int_{0}^{1} h\left(s^{-1}, E\right) M^{\prime}(s, \psi / \kappa) d s / s\left\|(x \kappa)^{* *}\right\|_{E} .
$$


Since $\beta^{\prime}(E)<1$, by Shimogaki's theorem (see [10, p. 187]),

$$
\left\|(x \kappa)^{* *}\right\|_{E} \leqslant \int_{0}^{1} h\left(s^{-1}, E\right) d s\|x \kappa\|_{E} .
$$

Hence $P_{\psi} \in\left[E_{\kappa, t}\right]$ and $\left\|P_{\psi}\right\| \leqslant C_{0}(E, \psi / \kappa) C_{0}(E$, id $)$.

(c) From the inequality $\kappa(t)\left(P_{\psi} x\right)(\delta(t)) \geqslant a_{\delta} P_{\psi \circ \delta / \kappa}(\kappa x \circ \delta)(t)$ a.e. on $I$ and from Theorem 2 of [15] we have our thesis.

As a consequence of Theorem 1 one obtains

Corollary 1 (Pavlov [18, Theorem 2] or [10, p. 194]; [19, Theorem 2]). Let $\kappa$ be a positive measurable and submultiplicative function on $\mathbf{R}_{+}$. If $E$ is a r.i. space on $\mathbf{R}_{+}$ such that $\beta^{\infty}(E)<1-q^{\infty}(\kappa)$ and $E$ has Fatou norm or $\beta^{\infty}(E)<1$, then $P_{1}=S_{1} \in$ $\left[E_{\kappa, t}\right]$.

Proposition 2. Let $E, \delta$ and $\kappa$ be as in Theorem 1. If $b_{\delta}<\infty, \beta^{\prime}(E)<p^{\prime}(\delta / \kappa)$ and $E$ has Fatou norm or $\beta^{\prime}(E)<1$, then

$$
E_{\kappa, \delta}^{(*)}=E_{\kappa, \delta}^{(* *)} \text {. }
$$

Proof. This follows immediately from Theorem 1.

THEOREM 2. Let $E$ be a r.i. space on $I$ and let $\psi: I \rightarrow \mathbf{R}_{+}, \delta: I \rightarrow I$ be increasing absolutely continuous functions and $\kappa: I \rightarrow \mathbf{R}_{+} a$ measurable function such that $\delta\left(0^{+}\right)=0$ and $p^{\prime}(\psi \circ \delta / \kappa)>0$.

(a) If $b_{\psi \circ \delta}<\infty$, E has Fatou norm and $\beta^{\prime}(E)<p^{l}(\psi \circ \delta / \kappa)$, then $S_{\psi} \in\left[E_{\kappa, \delta}\right]$ and $\left\|S_{\psi}\right\| \leqslant b_{\psi \circ \delta} C_{0}(E, \psi \circ \delta / \kappa)$.

(b) If $b_{\psi \circ \delta}<\infty$ and $\beta^{\prime}(E)<\min \left\{1, p^{l}(\psi \circ \delta / \kappa)\right\}$, then $S_{\psi} \in\left[E_{\kappa, \delta}\right]$ and $\left\|S_{\psi}\right\| \leqslant$ $b_{\psi \circ \delta} C_{0}(E, \psi \circ \delta / \kappa) C_{0}(E, \mathrm{id})$.

(b') If $\psi \circ \delta$ is a concave function on $I$ and $\beta^{\prime}(E)<p^{l}(\psi \circ \delta)$ then $S_{\psi} \in\left[E_{1, \delta}\right]$ and $\left\|S_{\psi}\right\| \leqslant \int_{0}^{1} h\left(s^{-1}, E\right) d M^{\prime}(s, \psi \circ \delta)$.

(c) If $S_{\psi} \in\left[E_{1, \delta}\right]$, then $\beta^{\prime}(E)<q^{l}(\psi \circ \delta)$.

Proof. Since $\left(S_{\psi} x\right)(\delta(t))=S_{\psi \circ \delta}(x \circ \delta)(t)$ a.e. on $I$, so $S_{\psi} \in\left[E_{\kappa, \delta}\right]$ if and only if $S_{\psi \circ \delta} \in\left[E_{\kappa, t}\right]$. Moreover,

$$
\left\|S_{\psi}\right\|_{\left[E_{\kappa, \delta}\right]}=\left\|S_{\psi \circ \delta}\right\|_{\left[E_{\kappa, t}\right]} .
$$

The thesis follows from the facts:

(a) and (b) from (21), the inequality $S_{\psi \circ \delta} \leqslant b_{\psi \circ \delta} P_{\psi \circ \delta}$ and Theorem 1;

(b') from the inequality

$$
\begin{aligned}
\left(S_{\psi \circ \delta} x\right)(t) & =[\psi \circ \delta(t)]^{-1} \int_{0}^{1} x(t s) d \psi(\delta(t s)) \\
& \leqslant[\psi \circ \delta(t)]^{-1} \int_{0}^{1} x *(t s) d \psi(\delta(t s))
\end{aligned}
$$

and Lemma 1;

(c) see [16, Theorem 4.4] (see also [14, Theorem 3.4]).

REMARKS 3. The reader will have no difficulty in formulating the results for the $Q_{\psi}$ and $T_{\psi}$ operators. 
4. Applying Theorems 1 and 2, and Proposition 1 [for $\kappa(t)=1$ and $\delta(t)=\tau^{-1}(t)$, $\tau$-from Example 1] we have boundedness of $P_{\psi}, Q_{\psi}, S_{\psi}$ and $T_{\psi}$ operators in $E(\mu)$ spaces. In particular case,

5. If $0<a_{\tau} \leqslant b_{\tau}<\infty, b_{\psi \circ \tau^{-1}}<\infty, 0<p^{l}\left(\psi \circ \tau^{-1}\right)=q^{l}\left(\psi \circ \tau^{-1}\right)$ and $E$ has Fatou norm, then

$$
\begin{gathered}
P_{\psi} \in[E(\mu)] \Leftrightarrow S_{\psi} \in[E(\mu)] \Leftrightarrow \beta^{\prime}(E)<p^{l}\left(\psi \circ \tau^{-1}\right), \\
Q_{\psi} \in[E(\mu)] \Leftrightarrow T_{\psi} \in[E(\mu)] \Leftrightarrow \alpha^{\prime}(E)>p^{\prime}\left(\psi \circ \tau^{-1}\right) .
\end{gathered}
$$

Corollary 2 (Andersen [1, Theorem 1]). Let $l=\infty, E$ have Fatou norm and $\tau(t)=t^{\sigma} / \sigma, \sigma>0$. From Remark 5 follows

$$
\begin{aligned}
& S_{a}=a P_{a} \in[E(\mu)] \Leftrightarrow \beta^{\infty}(E)<a / \sigma, \\
& T_{a}=a Q_{a} \in[E(\mu)] \Leftrightarrow \alpha^{\infty}(E)>a / \sigma .
\end{aligned}
$$

Corollary 3 (V. N. Sedov, See [11]). Let $0<l \leqslant \infty$ and $\tau(t)=\psi(t)$. If $p>1$ then

$$
\beta^{\prime}\left(L^{p}\right)=1 / p<1=p^{\prime}\left(\psi \circ \tau^{-1}\right),
$$

and by Theorem $2\left(\mathrm{~b}^{\prime}\right)$ we have

$$
S_{\psi} \in\left[L^{p}(\mu)\right] \text { and }\left\|S_{\psi}\right\|_{\left[L^{p}(\mu)\right]} \leqslant p /(p-1) .
$$

More generally, if $E$ is any r.i. space on I with the upper index $\beta^{\prime}(E)<1$, then by Theorem 2( $\left.\mathrm{b}^{\prime}\right)$ we have

$$
S_{\psi} \in[E(\mu)] \text { and }\left\|S_{\psi}\right\|_{[E(\mu)]} \leqslant \int_{0}^{1} h\left(s^{-1}, E\right) d s .
$$

Corollary 4 (Bennett [2, TheORem 6.2]-For $f(t)=t^{\alpha}, \alpha>0$ ). (i) Let $l=1$, $1 \leqslant p \leqslant \infty, \tau(t)=(1-\log t)^{-1}$ and $\psi(t)=(1-\log t)^{-1 / p} f\left((1-\log t)^{-1}\right)$, where $f>0$,

$$
0<a \leqslant u f^{\prime}(u) / f(u) \leqslant b<\infty \quad \forall 0<u \leqslant 1 .
$$

Since $\psi\left(\tau^{-1}(t)\right)=\psi(\exp (1-1 / t))=t^{1 / p} f(t)$, then

$$
p^{1}\left(\psi \circ \tau^{-1}\right)=1 / p+p^{1}(f)>1 / p+a>1 / p .
$$

By Theorem 2(a) we have $S_{\psi} \in\left[L^{p}(\mu)\right]$ and

$$
\left\|S_{\psi}\right\|_{\left[L^{p}(\mu)\right]} \leqslant(b+1 / p) \int_{0}^{1} M(s, f) d s / s=A,
$$

i.e.

$$
\begin{aligned}
\left\|f\left((1-\log t)^{-1}\right)^{-1} \int_{0}^{t} x(s) d s\right\|_{L^{p}(\nu)} & \\
& \leqslant \frac{A}{a+1 / p}\left\|t(1-\log t) f\left((1-\log t)^{-1}\right)^{-1} x(t)\right\|_{L^{p}(\nu)}
\end{aligned}
$$

where $L^{p}(\nu)=L^{p}((0,1), d t /[t(1-\log t)])$. 
(ii) Let $l=1,1 \leqslant p \leqslant \infty, \tau(t)=(1-\log t)^{-p}$ and

$$
\psi(t)=(1-\log t)^{-1} f\left((1-\log t)^{-1}\right)^{-1},
$$

where $f>0$,

$$
0<a \leqslant u f^{\prime}(u) / f(u) \leqslant b<1 \quad \forall 0<u \leqslant 1 .
$$

Since $\psi\left(\tau^{-1}(t)\right)=\psi\left(\exp \left(1-t^{-1 / p}\right)\right)=t^{1 / p} f\left(t^{1 / p}\right)^{-1}$, then

$$
q^{1}\left(\psi \circ \tau^{-1}\right)=\frac{1}{p}-\left(\frac{1}{p}\right) p^{1}(f)<\frac{1}{p}-\left(\frac{1}{p}\right) a<\frac{1}{p} .
$$

Hence we have $T_{\psi} \in\left[L^{p}(\mu)\right]$ and

$$
\left\|T_{\psi}\right\|_{\left[L^{p}(\mu)\right]} \leqslant \frac{1-a}{p} \int_{1}^{\infty} M\left(s^{-1 / p}, f\right) d s / s=B,
$$

i.e.

$$
\begin{aligned}
\| f\left((1-\log t)^{-1}\right) & \int_{t}^{1} x(s) d s \|_{L^{p}(\nu)} \\
& \leqslant \frac{B}{1-b}\left\|t(1-\log t) f\left((1-\log t)^{-1}\right) x(t)\right\|_{L^{p}(\nu)}
\end{aligned}
$$

REMARK 6. Heinig [9] proved the boundedness of Hardy $P_{1}=S_{1}$ operator in weighted spaces $L^{p}\left((0, l) ; w(x) x^{p-r-1} d x\right)$, where $w$ is a nonnegative, nonincreasing function defined on $(0, \infty), l=\sup \{t>0: w(t)>0\}, 0<r<\infty$ and $p \geqslant 1$. If $\tau^{\prime}(t)=w(t) t^{p-r-1}$ then, in order to apply Theorem 2, additional assumptions about $w, p$ and $r$ are needed; however, taking $\tau(t)=t$ and manipulating with the function $\psi$, we obtain Heinig's result (see [15, Corollary 3]). I suspect that this result is also obtained in the paper by Sedaev [20], which I only know from the review in RŽ Math. 1973, 8 \#681.

\section{REFERENCES}

1. K. F. Andersen, On Hardy's inequality and Laplace transforms in weighted rearrangement invariant spaces, Proc. Amer. Math. Soc. 39 (1973), 295-299. MR 47 \#3620.

2. C. Bennett, Intermediate spaces and the class $L \log ^{+} L$, Ark. Mat. 11 (1973), 215-228. MR 50 \#5452.

3. D. W. Boyd, The Hilbert transformation on rearrangement invariant spaces, Canad. J. Math. 19 (1967), 599-616. MR 35 \#3383.

4. Indices of function spaces and their relationship to interpolation, Canad. J. Math. 21 (1969), 1245-1254. MR 54 \#909.

5. Indices of Orlicz spaces, Pacific J. Math. 38 (1971), 315-323. MR 46 \#6008

6. P. L. Butzer and F. Fehèr, Generalized Hardy and Hardy-Littlewood inequalities in rearrangement invariant spaces, Comment. Math. Prace Mat. (Tomus Specialis in Honorum Ladislai Orlicz) 1 (1978), 41-64. MR 80c \#46037.

7. F. Fehèr, A note on weak-type interpolation and function spaces, Bull. London Math. Soc. 12 (1980), 443-451.

8. G. H. Hardy, J. E. Littlewood and G. Polya, Inequalities, Cambridge Univ. Press, New York, 1934.

9. H. P. Heinig, The Marcinkiewicz interpolation theorem extends to weighted spaces, Studia Math. 62 (1978), 163-168. MR 80f \#46028a.

10. S. G. Krein, Ju. I. Petunin and E. M. Semenov, Interpolation of linear operators, "Nauka”, Moscow, 1978. (Russian) MR 81 \# 46086. 
11. A. Kufner, Imbedding theorems for general Sobolev weight spaces, Ann. Scuola Norm. Sup. Pisa Cl. Sci. (4) 23 (1969), 373-386. MR 40 \#6253.

12. G. G. Lorentz, On the theory of spaces $\Lambda$, Pacific J. Math. 1 (1951), 411-429. MR 13, 470.

13. S. Lojasiewicz, An introduction to the theory of real variable functions, PWN, Warsaw, 1973. (Polish)

14. L. Maligranda, A generalization of the Shimogaki theorem, Studia Math. 71 (1981), 69-83.

15. __ Generalized Hardy inequalities in rearrangement invariant spaces, J. Math. Pures Appl. 59 (1980), 405-415.

16. Indices and interpolation, preprint.

17. B. Muckenhoupt, Hardy's inequality with weights, Studia Math. 44 (1972), 31-38. MR 47 \#16.

18. E. A. Pavlov, A generalization of the Hardy inequality, Collection of articles on applications of functional analysis, Voroneż. Technolog. Inst., Voroneż, 1975, pp. 109-114. (Russian) MR 56 \#12200.

19. Some properties of the Hardy-Littlewood operator, Mat. Zametki 26 (1979), 909-912. (Russian) MR 81d \#46028.

20. A. A. Sedaev, On Hardy's operator in weighted $L^{p}$ spaces, Sb. Trudov Aspirantov Mat. Fak. Voroneż, Un-ta, Voroneż, 1972, pp. 48-53. (Russian)

The Mathematical institute of the Polish academy of Sciences, Poznań Branch, Mielżýnskiego 27/29, 61-725 Poznań, Poland 